\title{
Functional Localization of the Frontal Eye Fields in the Common Marmoset Using Microstimulation
}

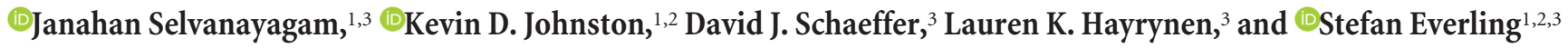 \\ ${ }^{1}$ Graduate Program in Neuroscience, ${ }^{2}$ Department of Physiology and Pharmacology, and ${ }^{3} \mathrm{Center}$ for Functional and Metabolic Mapping, Robarts Research \\ Institute, Western University, London, Ontario, Canada
}

The frontal eye field (FEF) is a critical region for the deployment of overt and covert spatial attention. Although investigations in the macaque continue to provide insight into the neural underpinnings of the FEF, due to its location within a sulcus, the macaque FEF is virtually inaccessible to electrophysiological techniques such as high-density and laminar recordings. With a largely lissencephalic cortex, the common marmoset (Callithrix jacchus) is a promising alternative primate model for studying FEF microcircuitry. Putative homologies have been established with the macaque FEF on the basis of cytoarchitecture and connectivity; however, physiological investigation in awake, behaving marmosets is necessary to physiologically locate this area. Here, we addressed this gap using intracortical microstimulation in a broad range of frontal cortical areas in three adult marmosets (two males, one female). We implanted marmosets with 96-channel Utah arrays and applied microstimulation trains while they freely viewed video clips. We evoked shortlatency fixed vector saccades at low currents $(<50 \mu \mathrm{A})$ in areas $45,8 \mathrm{aV}, 8 \mathrm{C}$, and $6 \mathrm{DR}$. We observed a topography of saccade direction and amplitude consistent with findings in macaques and humans: small saccades in ventrolateral FEF and large saccades combined with contralateral neck and shoulder movements encoded in dorsomedial FEF. Our data provide compelling evidence supporting homology between marmoset and macaque FEF and suggest that the marmoset is a useful primate model for investigating FEF microcircuitry and its contributions to oculomotor and cognitive functions.

Key words: common marmoset; frontal cortex; frontal eye fields; microstimulation; saccade

\section{Significance Statement}

The frontal eye field (FEF) is a critical cortical region for overt and covert spatial attention. The microcircuitry of this area remains poorly understood because in the macaque, the most commonly used model, it is embedded within a sulcus and is inaccessible to modern electrophysiological and imaging techniques. The common marmoset is a promising alternative primate model due to its lissencephalic cortex and potential for genetic manipulation. However, evidence for homologous cortical areas in this model remains limited and unclear. Here, we applied microstimulation in frontal cortical areas in marmosets to physiologically identify FEF. Our results provide compelling evidence for an FEF in the marmoset and suggest that the marmoset is a useful model for investigating FEF microcircuitry.

\section{Introduction}

Described originally by Ferrier (1875) as a cortical area in macaque monkeys where electrical stimulation elicited contralateral eye and head movements, the frontal eye fields (FEFs) in macaques and humans are now increasingly regarded as not only a

\footnotetext{
Received July 25, 2019; revised Sept. 23, 2019; accepted Sept. 26, 2019.

Author contributions: J.S., K.D.J., L.K.H., and S.E. performed research; J.S. and D.J.S. analyzed data; J.S. wrote the first draft of the paper; K.D.J. and S.E. designed research; K.D.J., D.J.S., L.K.H., and S.E. edited the paper.

This work was supported by the Canadian Institutes of Health Research (Grant FRN148365 to S.E.) and the Canada

First Research Excellence Fund to BrainsCAN. We thank C. Vander Tuin, N. Hague, W. Froese, and K. Faubert for expert technical and surgical assistance and care of the marmosets.

The authors declare no competing financial interests.

Correspondence should be addressed to Stefan Everling at severlin@uwo.ca.

https://doi.org/10.1523/JNEUROSCI.1786-19.2019

Copyright $\odot 2019$ the authors
}

motor area for saccades and head movements, but also as a critical region for the deployment of overt and covert spatial attention (Awh et al., 2006). Over the past 40 years, most of our knowledge regarding the neural processes in the FEF has come from experiments in awake, behaving macaque monkeys. In these Old World primates, FEF is defined as an area within the rostral bank and fundus of the arcuate sulcus from which electrical microstimulation evokes saccades at low currents $(<50 \mu \mathrm{A})$ (Bruce et al., 1985). Stimulation, recording, and pharmacological manipulation studies in trained macaque monkeys have and continue to provide critical insights into the neural processes in FEF that underlie saccade control and visual attention. However, the local FEF microcircuitry remains poorly understood because, due to its location within a sulcus, macaque FEF is virtually inaccessible to intralaminar recordings and manipulations. 
The New World common marmoset (Callithrix jacchus) is a promising alternative primate model for studying FEF microcircuitry. These small primates have a largely lissencephalic cortex and can be trained to perform saccadic eye movement tasks while head restrained (Mitchell et al., 2014; Johnston et al., 2018, 2019). A first step toward such experiments is the physiological identification of the FEF in marmosets. Existing evidence for the location of this area in this species, however, remains limited and unclear. An early marmoset study by Mott and colleagues (1910) reported that both eye and combined eye and head movements could be evoked by electrical stimulation at several frontal cortical sites. Subsequently, Blum and colleagues (1982) confirmed and extended these earlier results. They observed movements including ipsilateral and contralateral saccades, eye movements in all directions, and slow, drifting movements. It seems that these eye movements were evoked in areas $6 \mathrm{DC}, 6 \mathrm{DR}, 8 \mathrm{aD}$, and 46 , with no clear topography of direction or amplitude. Interpretation of these earlier studies is difficult, however, as the anesthetized preparations used most likely influenced the properties of the eye movements evoked (Robinson and Fuchs, 1969).

More recently, anatomical evidence has suggested that marmoset FEF lies within areas 45 and 8aV (Reser et al., 2013). Both areas have widespread connections with extrastriate visual areas and areas labeled FEF and FV by Collins et al. (2005), which may correspond to areas 45 and $8 \mathrm{aV}$ and contain clusters of neurons projecting to the SC, an area critical for the initiation of saccadic and orienting movements. Area $8 \mathrm{aV}$ in marmosets also contains large layer $\mathrm{V}$ pyramidal neurons, a cytoarchitectonic characteristic of macaque FEF (Stanton et al., 1989). Consistent with this notion, fMRI studies in marmosets have reported BOLD activation in areas 45 and $8 \mathrm{aV}$ in response to visual stimuli and saccades (Hung et al., 2015; Schaeffer et al., 2019), though a resting-state fMRI functional connectivity study found the strongest SC connectivity in area $8 \mathrm{aD}$, at the border of area 6DR (Ghahremani et al., 2017). The investigators proposed that this region either corresponded to the marmoset FEF or that it may encode largeamplitude saccades, whereas area $8 \mathrm{aV}$ may encode smallamplitude saccades.

Here, we set out to physiologically identify the marmoset FEF using the classical approach of intracortical electrical microstimulation (ICMS). We applied microstimulation trains via chronically implanted, 96-channel electrode arrays placed to target a broad range of frontal cortical areas in three awake marmosets. Our findings revealed a topography of contralateral saccade amplitude in marmoset frontal cortex similar to that observed in macaques (Bruce et al., 1985; Schall, 1997) and humans (Foerster, 1926), with small saccades being encoded in area 45 and lateral parts of area $8 \mathrm{aV}$, and larger saccades combined with contralateral neck and shoulder movements encoded in the medial posterior portion of area $8 \mathrm{aV}$, area $8 \mathrm{C}$, and area $6 \mathrm{DR}$.

\section{Materials and Methods}

\section{Subjects}

We obtained data from three adult common marmosets (Callithrix jacchus; M1 male, 17 months; M2 female, 20 months; M3 male, 23 months). All experimental procedures conducted were in accordance with the Canadian Council of Animal Care policy on the care and use of laboratory animals and a protocol approved by the Animal Care Committee of the University of Western Ontario Council on Animal Care. The animals were under the close supervision of university veterinarians.

Before the commencement of microstimulation experiments, each animal was acclimated to restraint in a custom primate chair (Johnston et al., 2018). Animals then underwent an aseptic surgical procedure under general anesthesia in which 96-channel Utah arrays $(4 \mathrm{~mm} \times 4 \mathrm{~mm}$; 1 mm electrode length; $400 \mu \mathrm{m}$ pitch; iridium oxide tips) were implanted in left frontal cortex. During this surgery, a microdrill was used to initially open $4 \mathrm{~mm}$ burr holes in the skull and were enlarged as necessary using a rongeur. Arrays were manually inserted; wires and connectors were fixed to the skull using dental adhesive (All-Bond; Bisco Dental Products). Once implanted, the array site was covered with silicone adhesive to seal the burr hole (Kwik Sil; World Precision Instruments). A screw hole was drilled into the skull on the opposite side to the location of the implanted array to place the ground screw. The ground wire of the array was then tightly wound around the base of the screw to ensure good electrical connection. A combination recording chamber/head holder (Johnston et al., 2018) was placed around the array and connectors and fixed in place using further layers of dental adhesive. Finally, a removable protective cap was placed on the chamber.

\section{Localizing the array}

To precisely determine array locations, high-resolution T2-weighted structural MRI images obtained before surgery were coregistered with CT scans obtained after surgery. The MRI images provided each marmoset's brain geometry with reference to the location of the skull, and the CT images allowed for localization of the skull and the array boundaries. By coregistering the skulls across the two modalities, the precise array-tobrain location was determined for each animal.

Presurgical MRIs were acquired using an 9.4 T, $31 \mathrm{~cm}$ horizontal bore magnet (Varian/Agilent), a Bruker BioSpec Avance III console with the software package Paravision-6, and a custom-built, high-performance $15-\mathrm{cm}$-diameter gradient coil with $400 \mathrm{mT} / \mathrm{m}$ maximum gradient strength (xMR) (Peterson et al., 2018). A geometrically optimize, eightchannel phased array receive coil was designed in-house, for SNR improvement and to allow for acceleration of the echo planar imaging of marmoset cohorts (Gilbert et al., 2019). Preamplifiers were located behind the animal and the receive coil was placed inside a quadrature birdcage coil ( $12 \mathrm{~cm}$ inner diameter) used for transmission. Before each imaging session, anesthesia was induced with ketamine hydrochloride at $20 \mathrm{mg} / \mathrm{kg}$. During scanning, marmosets were anesthetized with isoflurane and maintained at a level of $2 \%$ throughout the scan by means of inhalation. Oxygen flow rate was kept between 1.75 and $2.25 \mathrm{~L} / \mathrm{min}$ throughout the scan. Respiration, $\mathrm{SpO}_{2}$, and heart rate were continuously monitored and were observed to be within the normal range throughout the scans. Body temperature was also measured and recorded throughout, maintained using warm water circulating blankets, thermal insulation, and warmed air. All animals were head-fixed in stereotactic position using a custom-built MRI bed with ear bars, eye bars, and a palate bar housed within the anesthesia mask (Gilbert et al., 2019). All imaging was performed at the Centre for Functional and Metabolic Mapping at the University of Western Ontario. T2-weighted structural scans were acquired for each animal with the following parameters: $\mathrm{TR}=$ $5500 \mathrm{~ms}, \mathrm{TE}=53 \mathrm{~ms}$, field of view $=51.2 \times 51.2 \mathrm{~mm}$, matrix size $=$ $384 \times 384$, voxel size $=0.133 \times 0.133 \times 0.5 \mathrm{~mm}$, slices $=42$, band width $=50 \mathrm{kHz}$, GRAPPA acceleration factor: 2 .

CT scans were obtained on a micro-CT scanner (eXplore Locus Ultra; GR Healthcare Biosciences) after array implantation. Before the scan, marmosets were anesthetized with $15 \mathrm{mg} / \mathrm{kg}$ ketamine mixed with $0.025 \mathrm{mg} / \mathrm{kg}$ medetomidine. An X-ray tube potential of $120 \mathrm{kV}$ and a tube current of $20 \mathrm{~mA}$ were used for the scan, with the data acquired at $0.5^{\circ}$ angular increment over $360^{\circ}$, resulting in 1000 views. The resulting $\mathrm{CT}$ images were then reconstructed into $3 \mathrm{D}$ with isotropic voxel size of $0.154 \mathrm{~mm}$. Heart rate and $\mathrm{SpO}_{2}$ were monitored throughout the session. At the end of the scan, the injectable anesthetic was reversed with an intramuscular injection of $0.025 \mathrm{mg} / \mathrm{kg}$ Cepetor, Modern Veterinary Therapeutics.

The raw MRI and CT images were converted to NifTI format using dcm2niix (Li et al., 2016) and the MRIs were reoriented from the sphinx position using FSL software (Smith et al., 2004). Then, using FSL (FSLeyes nudge function), each animal's CT image was manually aligned to their MRI image based on the skull location, which allowed for colocalization of the array and brain surface. The array position from the CT image was determined by a hyperintensity concomitant with the metallic contacts contained within the array; this hyperintensity stood out against 
the lower intensities of the skull and surrounding tissues. A region of interest (ROI) was manually drawn within the array location for each animal to be displayed on the National Institutes of Health (NIH) marmoset brain atlas surface (Liu et al., 2018) for ease of viewing. The NIH marmoset brain atlas is an ultra-high-resolution ex vivo MRI image dataset that contains the locations of cytoarchitectonic boundaries (Liu et al., 2018). To determine the array location with reference to the cytoarchitectonic boundaries, we nonlinearly registered the NIH template brain to each marmoset's T2-weighted image using Advanced Normalization Tools (ANTs; Avants et al., 2011) software. The resultant transformation matrices were then applied to the cytoarchitectonic boundary image included with the NIH template brain atlas. The olfactory bulb was manually removed from the marmoset T2-weighted image of each animal before registration, as it was not included in the template image. As a result of the transformations, the template brain surface, the cytoarchitectonic boundaries, and the array location (ROI described above) could be rendered on each animals' individual native-space brain surface.

We expect that this alignment procedure will be useful for future studies seeking to transform a relatively large functional patch of interest (e.g., ventrolateral FEF) in template space (i.e., with reference to cytoarchitectonic boundaries) to individual animal's MRI native space to determine array implantation loci. Indeed, some variability in cytoarchitecture (or perhaps more importantly, functional architecture) can be expected to occur across individual animals. As such, highly specific localization, like that of individual electrode implantation may require additional mapping to optimize the localization of the saccade vector and amplitude of interest.

\section{Experimental design and statistical analysis}

Data collection. After recovery, we verified that electrode contacts were within the cortex by monitoring extracellular neural activity using the Open Ephys acquisition board (http://www.open-ephys.org) and digital head stages (Intan Technologies). Upon observing single- or multiunit activity at multiple sites in the array, we commenced microstimulation experiments.

Animals were head restrained in a custom primate chair (Johnston et al., 2018) mounted on a table in a sound attenuating chamber (Crist Instrument). A spout was placed at the monkey's mouth to deliver a viscous preferred reward of acacia gum. This was delivered via infusion pump (model NE-510; New Era Pump Systems). In each session, eye position was calibrated by rewarding $300-600 \mathrm{~ms}$ fixations on a marmoset face presented at one of five locations on the display monitor using the CORTEX real-time operating system (National Institute of Mental Health-NIH). Faces were presented at the display center, at $6^{\circ}$ to the right and left of center and at $6^{\circ}$ directly above and below center. All stimuli were presented on a CRT monitor (ViewSonic Optiquest Q115, $76 \mathrm{~Hz}$ noninterlaced, $1600 \times 1280$ resolution).

Monkeys freely viewed short, repeating video clips to sustain their alertness while we applied manually triggered microstimulation trains. Monkeys were intermittently rewarded at random time intervals to maintain their interest. Microstimulation trains were delivered using the Intan RHS2000 stimulation/recording controller system and digital stimulation/recording head stages (Intan Technologies). Stimulation trains consisted of $0.2-0.3 \mathrm{~ms}$ biphasic current pulses delivered at $300 \mathrm{~Hz}$ for a duration of 100-400 ms at current amplitudes varying between 5 and $300 \mu \mathrm{A}$. At sites where skeletomotor or saccadic responses were evoked, we performed a current series to determine thresholds. The threshold was defined as the minimum current at which a given response was evoked on $50 \%$ of stimulation trials. Skeletomotor responses were observed and recorded manually by two researchers. Eye position was digitally recorded at $1 \mathrm{kHz}$ via video tracking of the left pupil (EyeLink 1000; SR Research).

Data analysis. Analysis was performed with custom python code. Eye velocity (visual degrees per second) was obtained by smoothing and numerical differentiation. Saccades were defined as horizontal or vertical eye velocity exceeding $30^{\circ} \mathrm{s}$. Blinks were defined as the radial eye velocity exceeding $1500 \%$ s. Slow eye movements were manually identified by inspection of the eye traces.
Because we did not require marmosets to fixate during stimulation, saccades after stimulation could be spontaneous. A bootstrap analysis was used to quantitatively determine whether saccades were more probable after stimulation than at any other time during a session. In a single session, 60-80 trains were delivered at a single site holding stimulation parameters constant over a $2 \mathrm{~min}$ period. Stimulation onset times were shuffled (time points were randomly sampled without replacement with millisecond resolution over the duration of the session) and the probability of a saccade occurring in a $200 \mathrm{~ms}$ window the selected time points was computed. This was repeated 1000 times for each session to obtain a distribution of probabilities of saccade occurrence. The percentile rank of the probability of stimulation evoking a saccade with respect to this distribution was computed; the 95 th percentile marked the $5 \%$ significance criterion indicating a session where stimulation significantly increased the probability of saccade occurrence.

\section{Results}

\section{Evoked skeletomotor and oculomotor responses}

Array locations were confirmed using CT scans obtained after the surgery, which were coregistered with MR scans obtained before the surgery (Fig. 1A). Microstimulation was conducted at 288 sites across three marmosets. We observed a range of skeletomotor and oculomotor responses across the frontal cortex (Fig. $1 B, C$ ).

At the most posterior sites, we observed primarily single joint movements with a gross mediolateral topography. We observed hindlimb movements (leg, foot, toes) most medially, followed by forelimb (arm, hand, finger) and facial movements (eyelid, ear, nose, jaw) most laterally, an organization characteristic of primary motor cortex (area 4) (Burish et al., 2008; Wakabayashi et al., 2018). Anterior to this, we observed overlapping representation of forelimb, facial, shoulder, and neck musculature with no obvious organization, similar to that observed in the marmoset premotor cortex (area 6) (Burish et al., 2008; cf. Wakabayashi et al., 2018).

We elicited saccades at 61 sites across three marmosets (Fig. $1 C)$. At six sites on the border of area $6 \mathrm{DC}$ and $6 \mathrm{M}$, we observed goal directed saccades characteristic of the supplementary eye fields (SEFs), albeit at long latencies (70-110 ms) and high currents $(200 \mu \mathrm{A})$ (Fig. 2A). At three sites in area 46D and the anterior portion of area $8 \mathrm{aD}$, we elicited saccades with no clear pattern at long latencies (75-90 ms) and high currents $(300 \mu \mathrm{A})$ (Fig. $2 B$ ). Saccades evoked from these sites were mostly directed to the hemifield contralateral to the stimulated site, though some saccades directed to the ipsilateral hemifield were observed.

We elicited fixed vector saccades at 52 sites across areas 6DR, $8 \mathrm{C}, 8 \mathrm{aV}$, and 45 . Mean saccade vectors are plotted in Figure $1 C$. Representative saccade traces are plotted in Figure 3. In areas $6 \mathrm{DR}, 8 \mathrm{C}$, and the medial portion of $8 \mathrm{aV}$, we observed larger saccades often coupled with shoulder, neck, and ear movements, with the most common response being a shoulder rotation that resembled orienting toward contralateral side. In area 45 and the lateral portion of area $8 \mathrm{aV}$, we observed smaller saccades with no visible skeletomotor responses. Slow eye movements could be elicited at five sites in areas 6DR and 8C.

\section{Saccade thresholds and latencies}

At sites where we observed fixed vector saccades, we conducted current series to determine thresholds and characterize any current-related changes in saccade metrics. Current series from five representative sites are shown in Figure 4, $A-E$. Thresholds were defined as the minimum current at which saccades could be evoked $50 \%$ of the time (Fig. $4 G$ ). Thresholds ranged from 12 to $300 \mu \mathrm{A}$. Saccades were evoked at low thresholds $(<50 \mu \mathrm{A})$ at 35 of the 52 sites from which we were able to evoke fixed vector saccades (Fig. 1D). Saccade metrics were computed at the minimum current at which saccades could be evoked $75 \%$ of the time. 


\section{Marmoset 1}

A

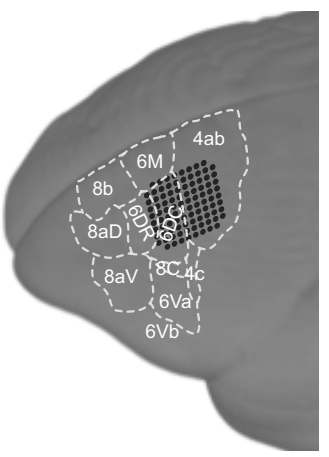

Marmoset 2

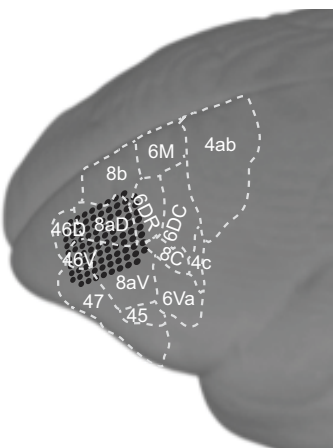

Marmoset 3
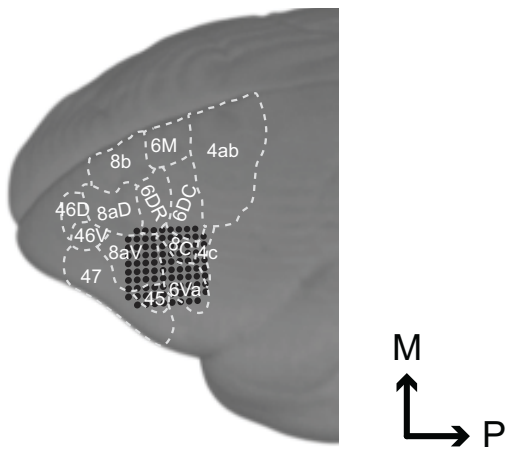

B

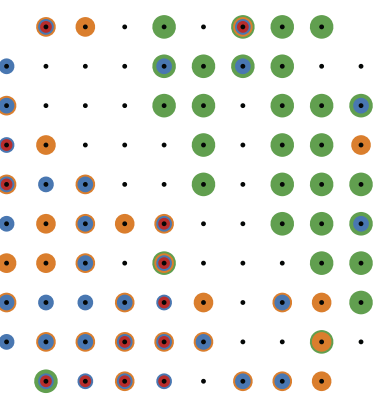

C
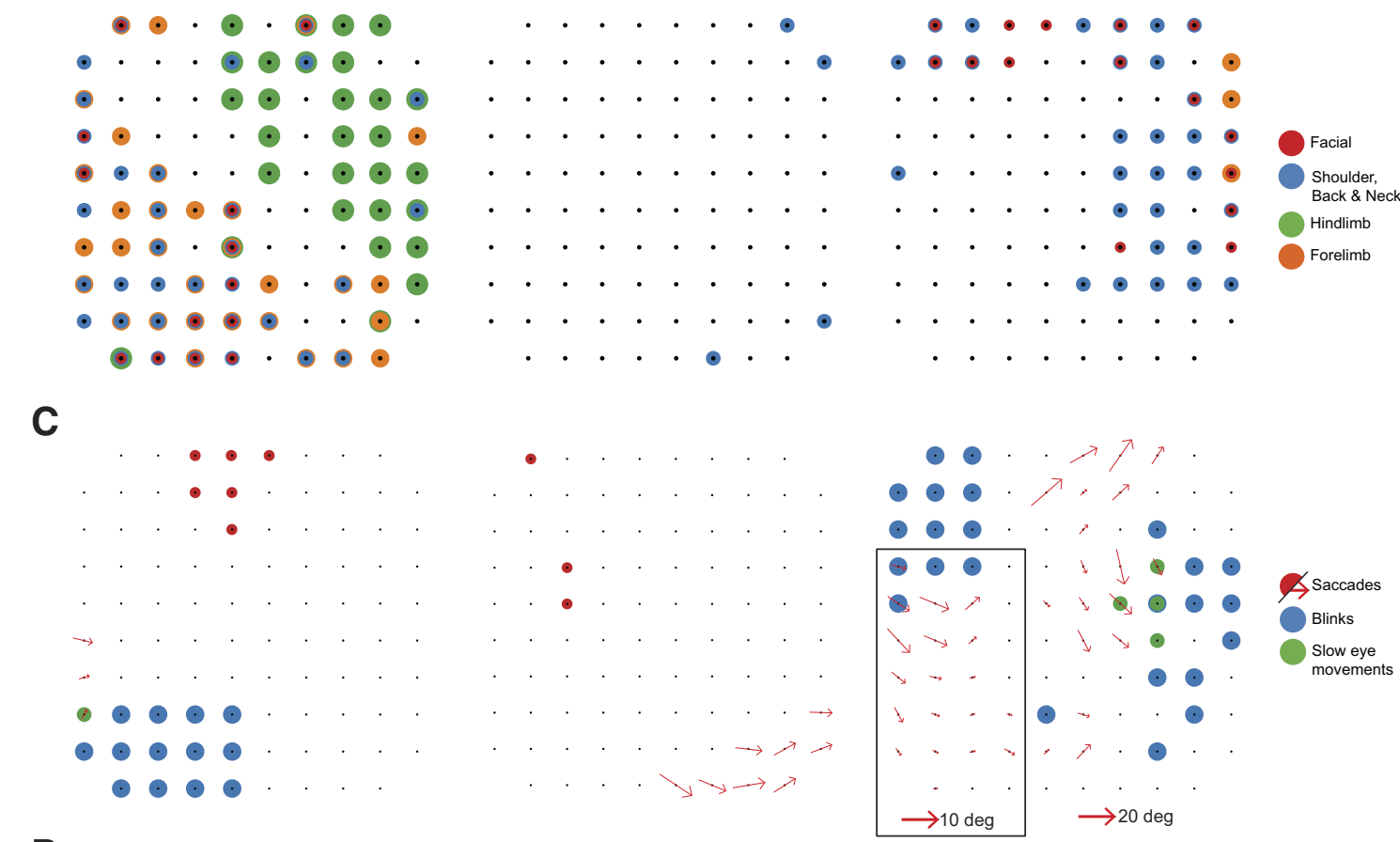

D

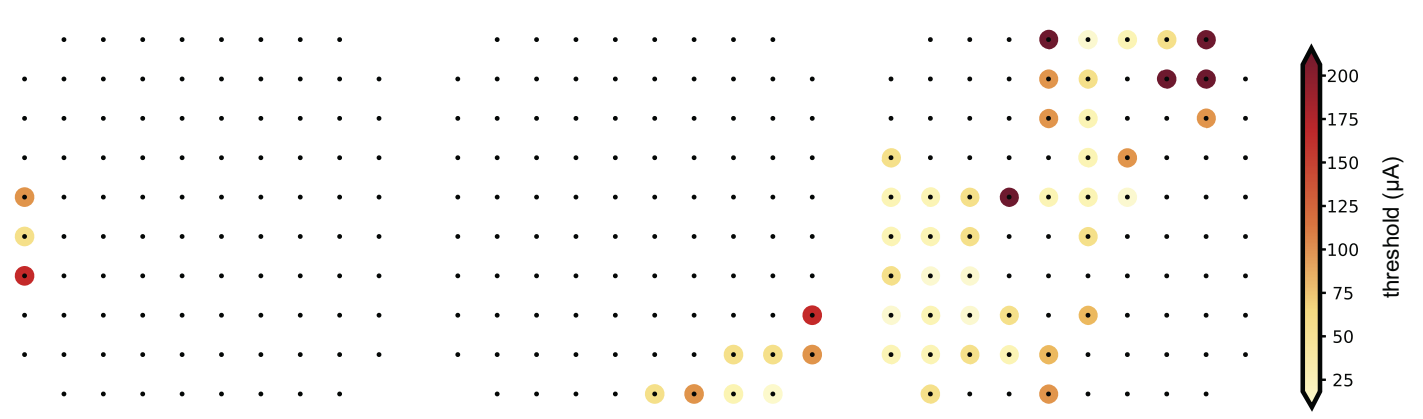

Figure 1. Evoked motor responses. A, Array locations in each marmoset reconstructed using MR and (T images (see "Localizing the array" section). B, Pattern of evoked skeletomotor responses in each marmoset. $C$, Pattern of evoked oculomotor responses in each marmoset. At sites where fixed vector saccades were observed, mean saccade vector is plotted. Mean saccade vectors were computed at the minimum current where saccades are evoked at least $75 \%$ of the time. Inset shows small saccade vectors at $2 \times$ scale for Marmoset 3 . D, Thresholds for saccades at sites where saccades were evoked at currents $\leq 300 \mu \mathrm{A}$.

Each site had a stereotypical saccade latency, though we found no systematic variation in saccade latency with respect to site coordinates nor any other saccade metrics. Saccade latencies ranged from 25 to $85 \mathrm{~ms}$, with the majority falling in the range between 40 and $60 \mathrm{~ms}$ (Fig. $4 \mathrm{H}$ ). Saccade latencies were generally longer and more variable near the current threshold for a given site.
When using high currents well above threshold (200-300 $\mu \mathrm{A})$, uniformly short saccade latencies were observed (15-45 ms).

\section{Topography of evoked saccades}

Evoked saccades were directed contralateral to the stimulated hemisphere and mostly fixed vector (Figs. $1 C, 3, A-E$ ), exhibiting 

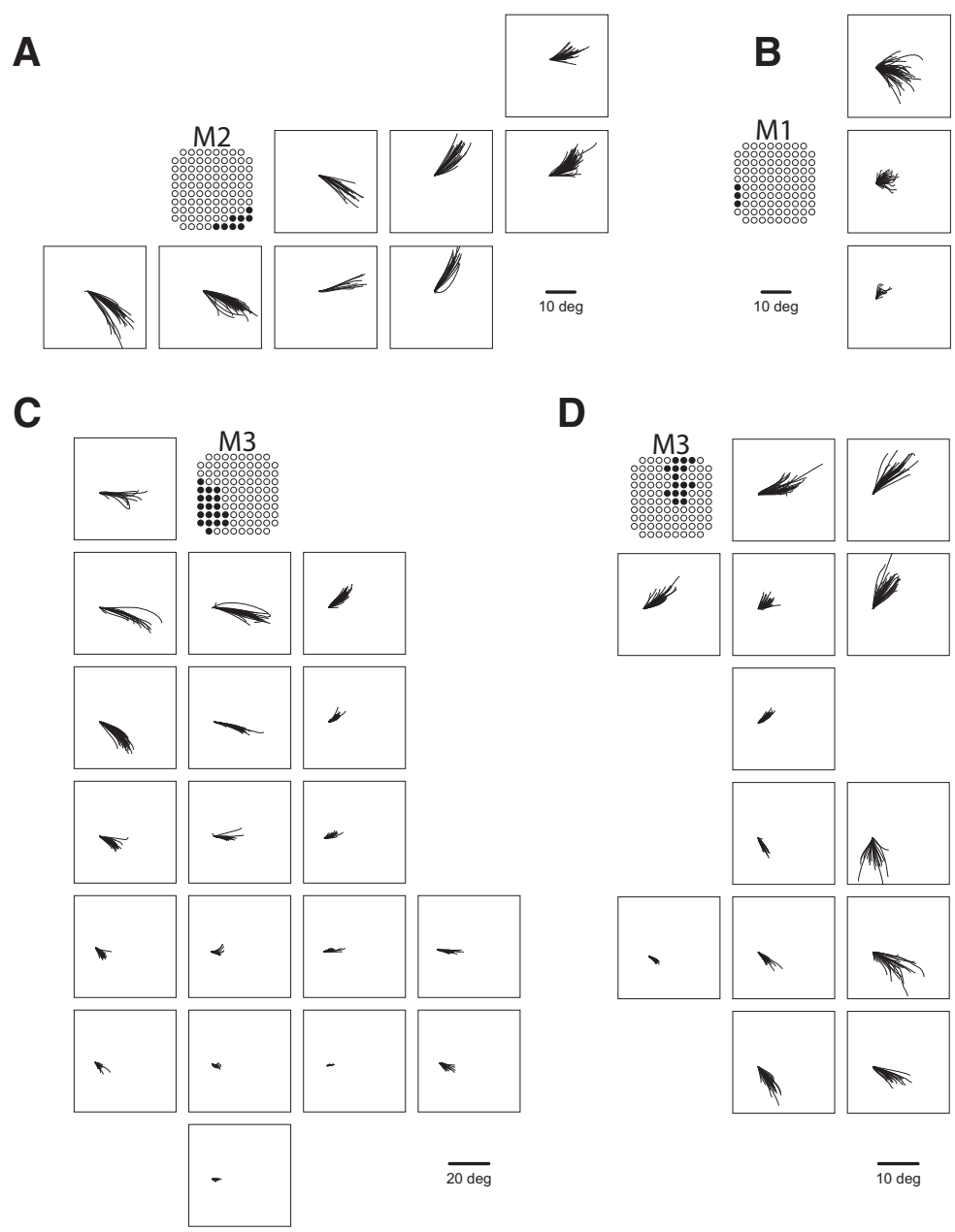

D

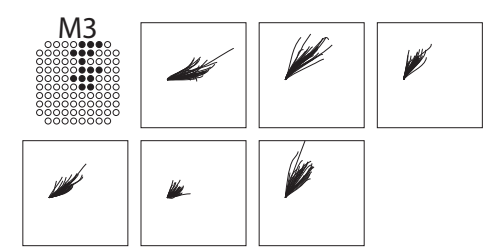

Figure 2. Saccades evoked in FEF sites. Representative traces for fixed vector saccades in Marmoset 2 (A), Marmoset 1 ( $\boldsymbol{B})$, and Marmoset $3(\boldsymbol{C}, \boldsymbol{D})$.
A
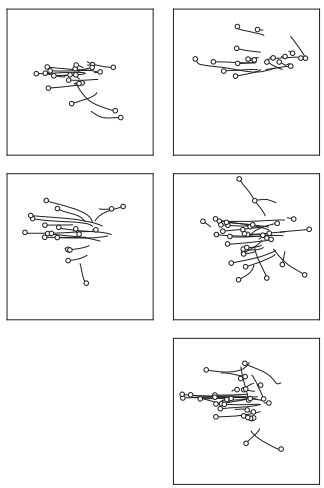
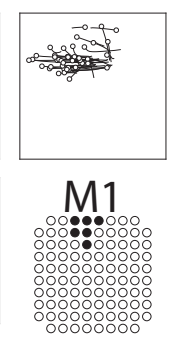

$\overline{10 \mathrm{deg}}$
B
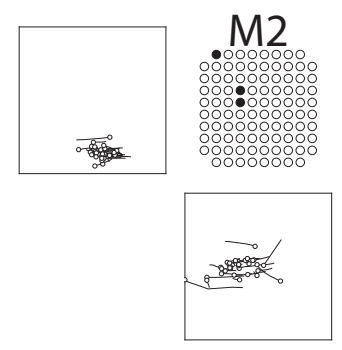

$\overline{10 \mathrm{deg}}$

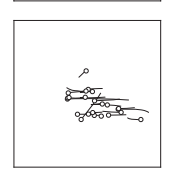

Figure 3. Saccades evoked in non-FEF sites. Shown are representative traces for goal-directed saccades from dorsomedial sites in Marmoset $1(\boldsymbol{A})$ and saccades from rostral sites in Marmoset $2(\boldsymbol{B})$. Open circles indicate eye position at saccade onset.

keys (Heiney and Blazquez, 2011). 90\% of initial eye positions fell within the following ranges for each marmoset: Marmoset 1: -13.6 to 12.4 abscissa, -10.7 to 11.4 ordinate; Marmoset 2: -12.7 to $15.7 \mathrm{ab}-$ scissa, -11.7 to 9.6 ordinate; Marmoset 3: -12.9 to 12.7 abscissa, -18.5 to 14.3 ordinate. Amplitude decreased progressively from medial (large saccades; $>20$ visual degrees) to lateral (small saccades; $<2$ visual degrees) sites. Direction varied systematically from upper visual field at posterior medial sites to lower visual field at anterior lateral sites.

\section{Staircase saccades}

At a subset of sites from which saccades were evoked, we additionally observed staircases of multiple saccades. To investigate this further, we applied stimulation trains of increasing duration at these sites and found that the number of saccades increased as a function of train duration at the majority of these sites (12/15). A representative site is depicted in Figure 5. Staircases consisted of two to five consecutive saccades with consistent amplitudes and directions, in many cases ultimately driving the eye to the extent of its oculomotor range. At a given site, consecutive saccades occurred at fixed intervals. The intersaccadic interval ranged from 70 to $120 \mathrm{~ms}$ across sites and we observed no systematic variation in intersaccadic interval with respect to site coordinates nor any other saccade metrics.

\section{Slow eye movements}

Posterior to where we evoked saccades, in areas 6DR and $8 \mathrm{C}$ (Fig. 1C), we were able to elicit slow eye movements. These eye movements often followed a saccade and continued until stimulation ended, at which point they stopped abruptly (Fig. $6 A$ for a representative site). Slow eye movement duration ranged from 50 to 75 ms, varying as a function of stimulation site. Although the direction of these movements tended to be consistent at a site, the velocity increased as a function of stimulation current intensity, consistent with what has been observed in the smooth pursuit region of the FEF in macaques (Fig. $6 B$ for a current series at a representative site) (Gottlieb et al., 1993).

relatively consistent directions and amplitudes independent of the initial eye position. Although we did not systematically vary initial eye positions, the fact that marmosets were allowed to freely direct their gaze across video clips on the display monitor during experimental sessions ensured a wide range of initial eye positions at the time of microstimulation onset. Most initial eye positions fell within a $13^{\circ}$ range similar to observations elsewhere in marmosets (Mitchell et al., 2014) and other New World mon-
Radial eye velocity ranged from 10 to 200 visual degrees/s, varying as a function of stimulation site and current intensity.

\section{Effects of initial gaze position}

Although evoked saccades were mostly fixed vector, an effect of initial gaze position was observed at some sites. At those sites, saccades tended to be of greater amplitude if the gaze position at the time of stimulus onset was within the hemifield ipsilateral to 

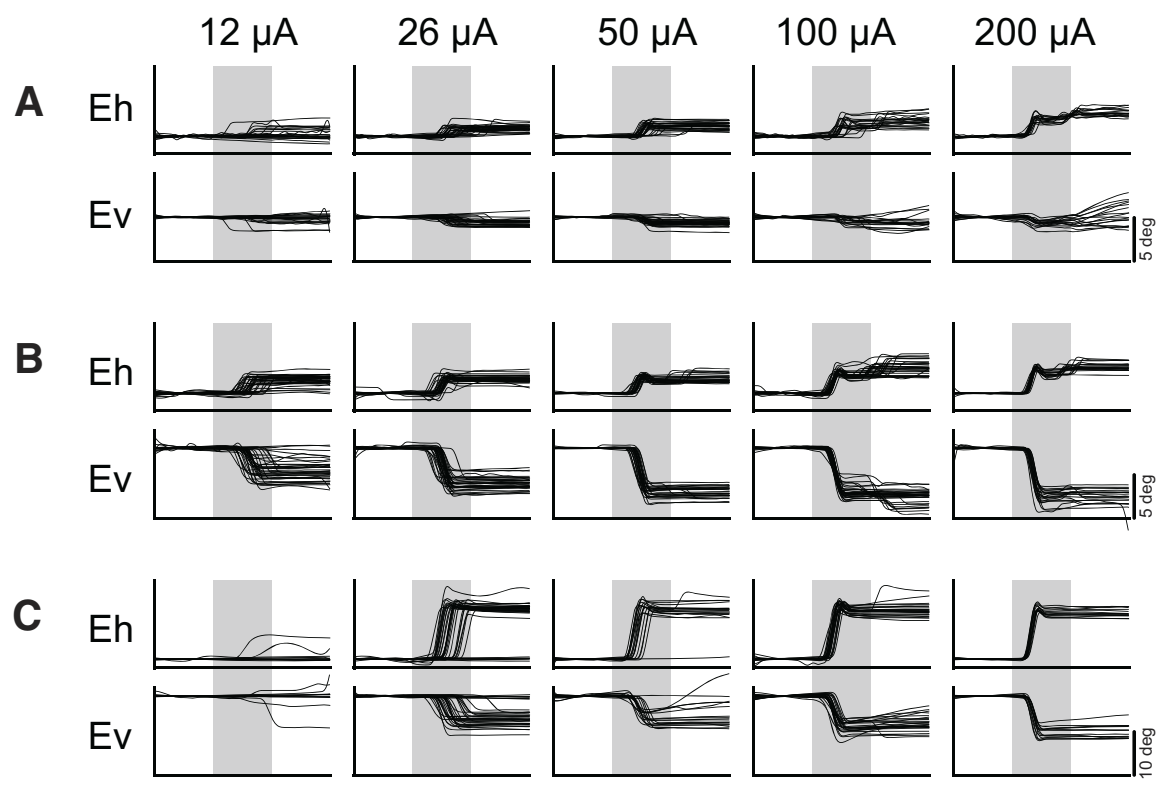

$\mathbf{F}$
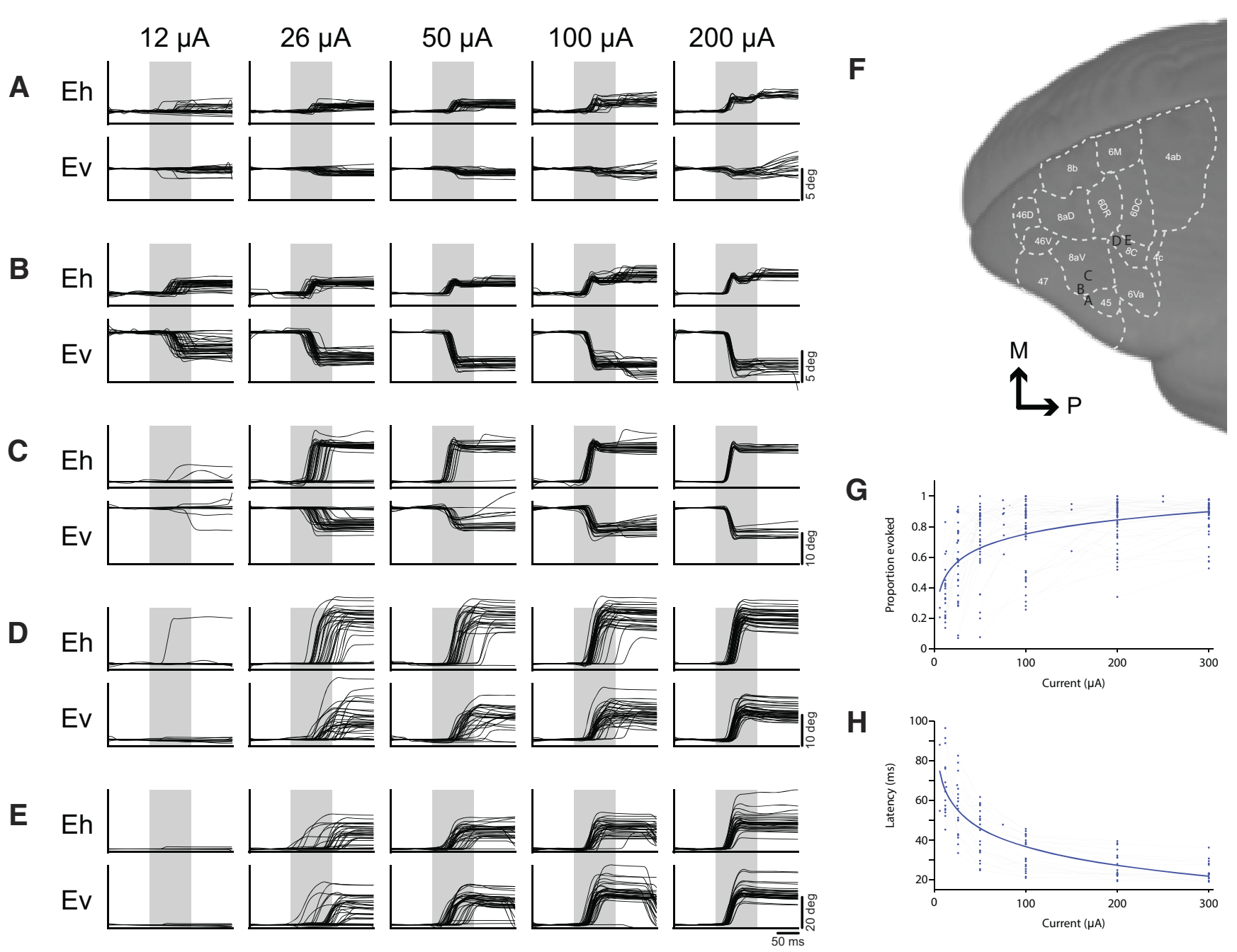

Figure 4. Current series at representative saccade sites. Current series at a representative small $(\boldsymbol{A}-\boldsymbol{C})$ and large $(\boldsymbol{D}, \boldsymbol{E})$ saccade sites. Baseline correction was applied by subtracting the mean gaze position during a $100 \mathrm{~ms}$ period preceding stimulation onset in the horizontal and vertical components separately. Gray bars indicate stimulation train duration. Location of array sites for series in $\boldsymbol{A}-\boldsymbol{E}$ are shown in $\boldsymbol{F}$. G, Effect of current on proportion of saccades evoked at all FEF sites in Marmoset 3. $\boldsymbol{H}$, Effect of current on saccade latency at low threshold ( $<50 \mu \mathrm{A})$ sites in Marmoset 3.
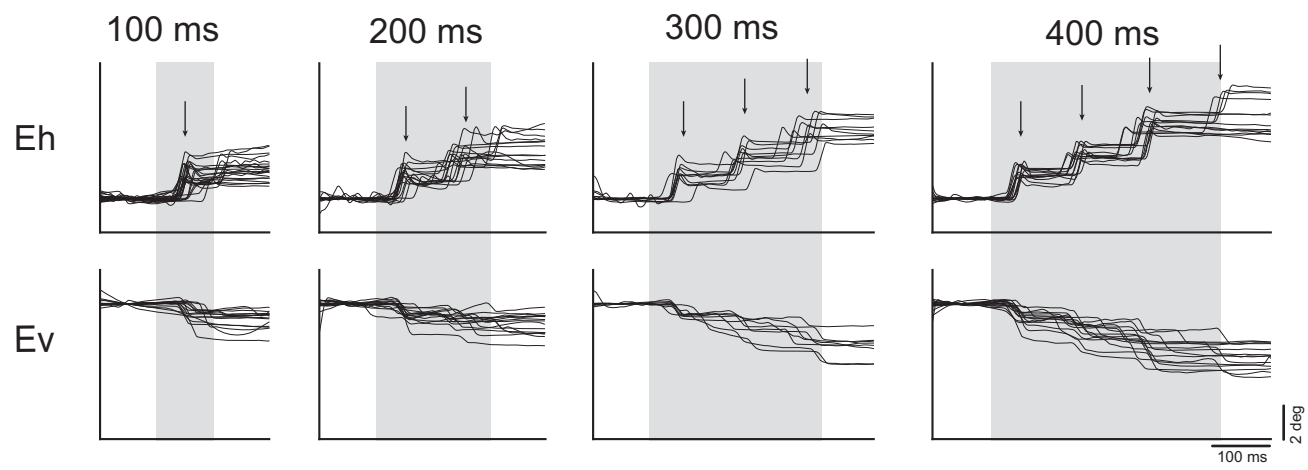

Figure 5. Current series at a representative site with staircase saccades. Arrows indicate median saccade onset latency. Baseline correction was applied by subtracting the mean gaze position during a $100 \mathrm{~ms}$ period preceding stimulation onset in the horizontal and vertical components separately. Gray bars indicate stimulation train duration.

the stimulated hemisphere. Further, the probability of evoking a saccade was lower if the initial eye position was within the hemifield contralateral to the stimulated hemisphere.

We quantified the magnitude of the effect of initial eye position at each site by computing the linear regression of the difference in final eye position as a function of the initial eye position separately for horizontal $\left(K_{\mathrm{h}}\right)$ and vertical $\left(K_{\mathrm{v}}\right)$ com- ponents of evoked saccades at these sites (Russo and Bruce, 1993). Correlation coefficients of 0 would be expected for sites at which the saccade vector did not change with varying initial eye positions (i.e., strictly fixed-vector saccades), whereas coefficients of -1 would be expected for sites at which evoked saccades terminated at the same eye position regardless of initial eye position (i.e., goal-directed saccades). An example 
A

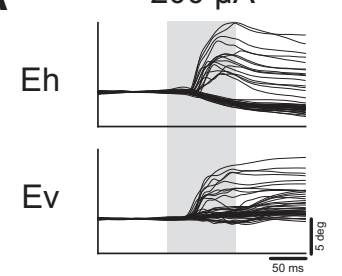

B

Eh

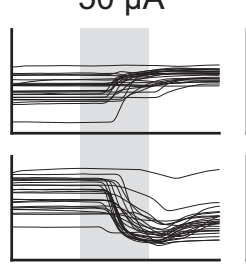

$76 \mu \mathrm{A}$

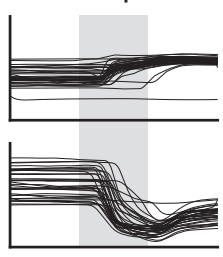

$100 \mu \mathrm{A}$

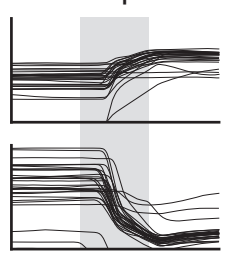

$200 \mu \mathrm{A}$

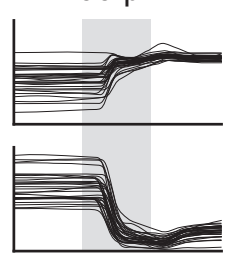

$300 \mu \mathrm{A}$

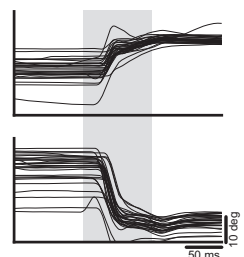

Figure 6. Evoked slow eye movements. $\boldsymbol{A}$, Slow eye movement site at $200 \mu \mathrm{A}$ from Marmoset 1 Baseline correction was applied by subtracting the mean gaze position during a $100 \mathrm{~ms}$ period preceding stimulation onset in the horizontal and vertical components separately. $\boldsymbol{B}$, Current series from a slow eye movement site in Marmoset 3 . Gray bars indicate stimulation train duration.

A
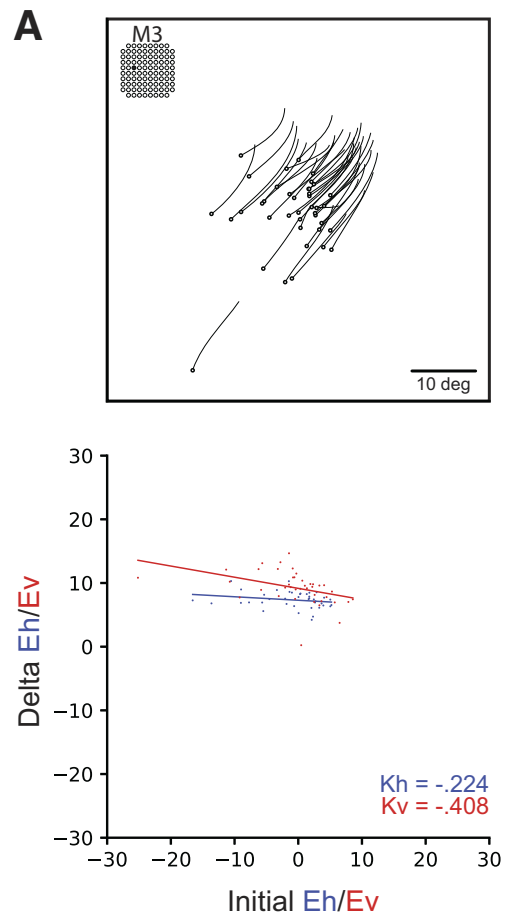

D

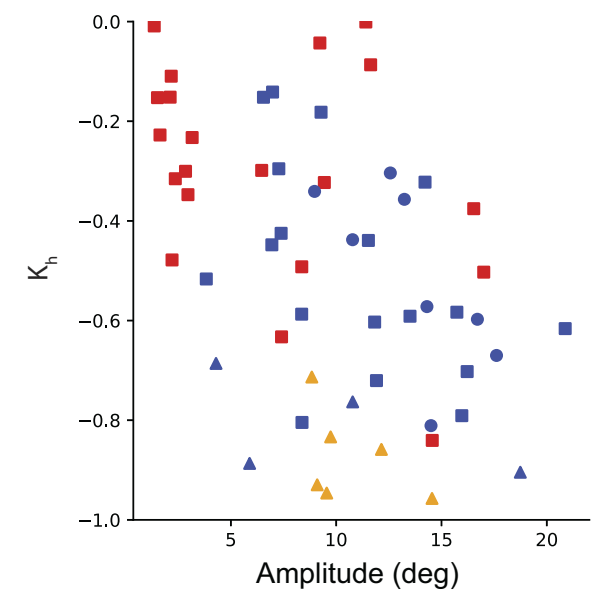

B
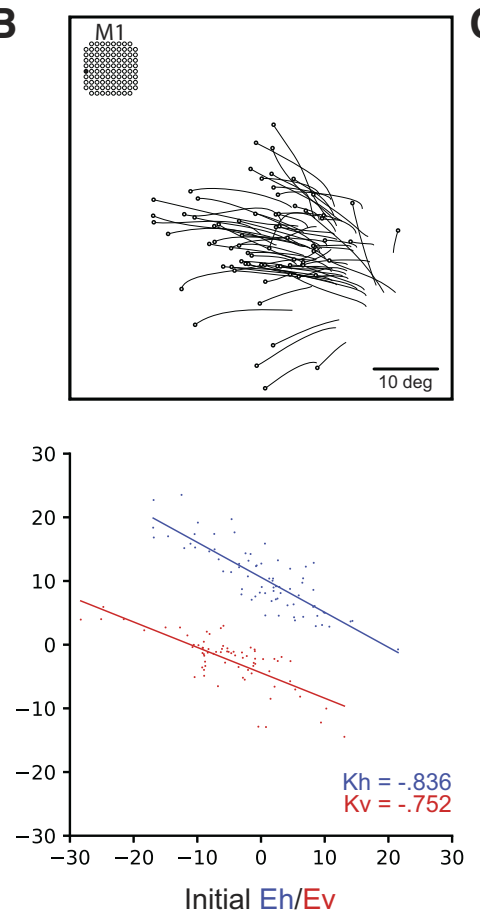
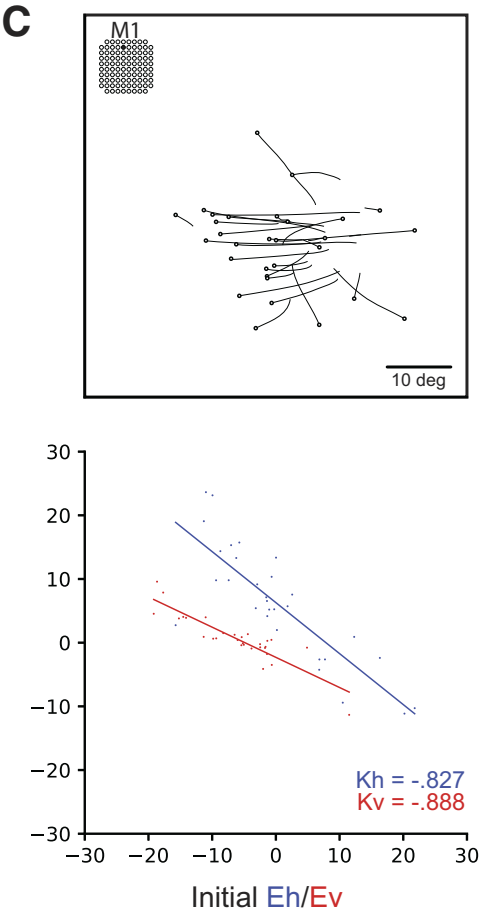

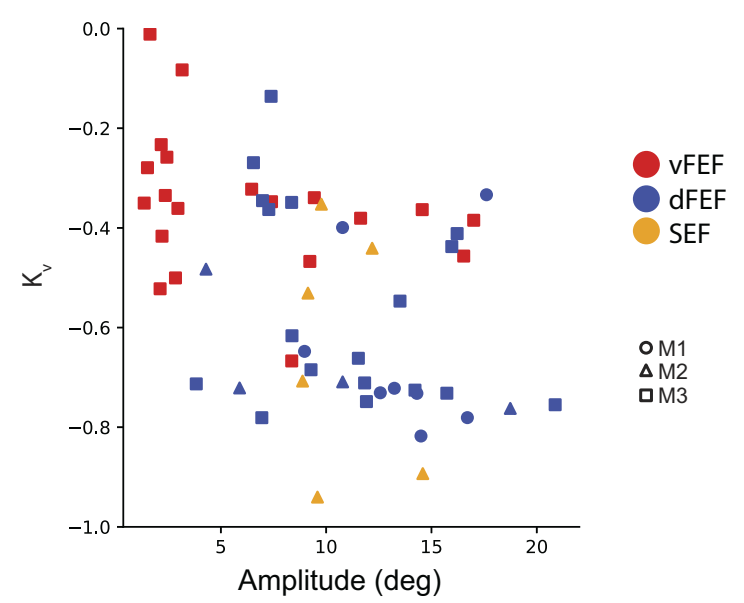

Figure 7. Effect of initial eye position. Saccade traces (above) and effect of initial position on delta (below) for representative sites from vFEF ( $\boldsymbol{A}$ ), dFEF ( $\boldsymbol{B})$, and SEF ( $\boldsymbol{C}$ ). Open circles indicate eye position at saccade onset. $\boldsymbol{D}$, Relationship between $K_{\mathrm{h}}$ and $K_{\mathrm{v}}$ values (correlation coefficients from effect of initial eye position analysis) and amplitude across all sites. More negative values indicate a greater effect of initial eye position.

of this is shown for representative sites from FEF (Fig. $7 A, B$ ) and SEF (Fig. 7C).

Sites in FEF were mostly fixed vector; however, an effect of initial eye position was observed which increased in magnitude with the mean amplitude of saccades evoked at that site (Fig. 7D).
This corresponds with the eye position terminating at the edge of the orbit for very large saccades. In contrast, in SEF sites, mostly convergent saccades were observed with correlation coefficients close to -1 and saccades converging on locations well within the oculomotor range of the animal. 


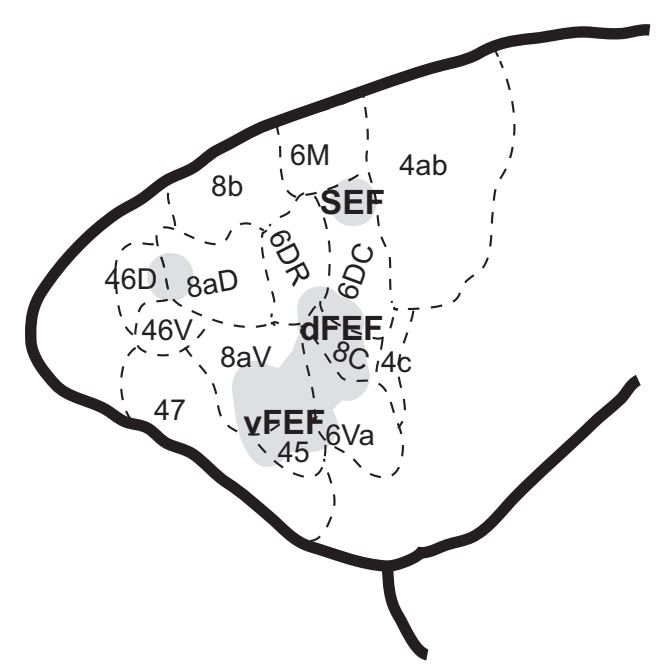

Figure 8. Schematic representation of cortical eye fields in marmoset frontal cortex.

\section{Discussion}

The common marmoset is a promising model for investigating the microcircuitry of the FEF (Mitchell and Leopold, 2015). The location of the FEF in marmosets, however, remains controversial. To address this, we systematically applied ICMS to marmoset frontal cortex through chronically implanted electrode arrays to investigate the oculomotor and skeletomotor responses evoked in this region (see Fig. 8 for a schematic summary). We observed patterns of skeletomotor responses consistent with previous ICMS investigations of marmoset motor and premotor cortex (Burish et al., 2008; Wakabayashi et al., 2018). Anterior to these motor areas, we observed a suite of oculomotor responses across frontal cortex that we propose correspond to three cortical eye fields. ICMS in area 45 and in the lateral part of area $8 \mathrm{aD}$ evoked small, contraversive saccades at very low currents, consistent with the properties of the ventrolateral FEF ( $\mathrm{vFEF}$ ) in macaques (Bruce et al., 1985). In areas 6DR, 6DC, 8C, and medial $8 \mathrm{aV}$, ICMS evoked larger saccades that were often associated with shoulder, neck, and ear movements. This is consistent with ICMS experiments in dorsomedial macaque FEF (dFEF) (Elsley et al., 2007; Corneil et al., 2010). We also observed goal-oriented saccades characteristic of the supplementary eye field (SEF) at dorsomedial sites. In prefrontal areas 46 and anterior $8 \mathrm{aD}$, ICMS elicited saccades with no consistent organization of direction or amplitude. These findings are consistent with the organization of FEF and SEF in macaques (Robinson and Fuchs, 1969; Bruce et al., 1985; Schlag and Schlag-Rey, 1987; Gottlieb et al., 1993; Russo and Bruce, 1993; Knight and Fuchs, 2007).

A characteristic feature of the FEF observed in macaque ICMS experiments is the ability to evoke short-latency, fixed-vector saccades at low currents. Although the threshold to evoke saccades can be as high as $2 \mathrm{~mA}$ in frontal cortex (Robinson and Fuchs, 1969), FEF is defined in macaque as the restricted region in which thresholds are $<50 \mu \mathrm{A}$ (Bruce et al., 1985). Here, we observed a large number of sites with thresholds $<50 \mu \mathrm{A}$, with a lower bound of $12 \mu \mathrm{A}$, similar to the $10 \mu \mathrm{A}$ observed in macaque (Bruce et al., 1985). This is despite the limitations of fixed-length chronic electrode arrays that did not allow us optimally target layer $\mathrm{V}$ output neurons and in contrast to previous reports of higher thresholds in marmoset motor cortex compared with macaques (Burish et al., 2008). However, saccade latencies were slightly longer than those observed in macaques. We found a range of 25-85 ms compared with 20-60 ms observed by Bruce and colleagues (1985) at near threshold currents, and 15-45 ms compared with 15-25 ms by Robinson and Fuchs (1969) at higher currents. It has been proposed that longer-latency saccades are evoked through an indirect route (e.g., superior colliculus), whereas shorter-latency saccades are evoked by recruiting neurons that project directly to the brainstem (Bruce et al., 1985). Investigations using single-unit recordings in the marmoset FEF and studies investigating the connectivity of marmoset FEF and brainstem oculomotor nuclei should provide insight into these differences.

In macaque FEF, saccades evoked by ICMS are fixed-vector with little variability in amplitude and direction (Robinson and Fuchs, 1969; Bruce et al., 1985). Although saccades evoked here were predominantly fixed vector, some effects of initial gaze position were observed in which saccades were larger when the initial gaze position was in the hemifield ipsilateral to the site of stimulation. Similar observations have been made in macaque FEF (Robinson and Fuchs, 1969; Russo and Bruce, 1993), in which the magnitude of this effect is greater for larger saccades. However, this effect is greater here than previously observed with macaques. This may be a result of the eye being driven to the edge of the oculomotor range. In marmosets, this is limited to $\sim 12^{\circ}$ compared with $30^{\circ}$ in the macaque (Tomlinson and Bahra, 1986; Heiney and Blazquez, 2011; Mitchell et al., 2014). Head restraint also prevents marmosets from using head movements to shift gaze, which they depend on to a greater extent than larger primates (Mitchell et al., 2014). Investigations in head-unrestrained marmosets would clarify these differences.

Previous studies of macaque FEF have revealed a topographic representation of saccade amplitude and direction. Bruce and colleagues (1985) demonstrated a mediolateral gradient in which large saccades were evoked medially and small saccades laterally. We observed a similar organization of saccade amplitude in marmosets, with small saccades being elicited in areas 45 and lateral area $8 \mathrm{aV}(\mathrm{vFEF})$ and larger saccades being evoked in areas 6DR, 6C, 8C, and medial 8aV (dFEF). Bruce and colleagues (1985) observed systematic changes in saccade direction with small advances along the depth of the arcuate sulcus in macaques, though they often encountered disruptions and reversals of direction. We observed a rostrocaudal organization of saccade direction in marmosets in which direction gradually changed from lower to upper visual field, though there were occasional direction reversals. Assuming that frontal cortex in marmoset is approximately a flattened version of that in macaque, the rostrocaudal axis would correspond approximately to traversing the depth of the arcuate sulcus from lip to fundus in macaques. We additionally observed a more continuous mediolateral organization of saccade direction, such that the upper visual field was represented medially. This organization is difficult to observe in the macaque FEF due to its more complex morphology. Savaki and colleagues (2015) investigated this using ${ }^{14} \mathrm{C}$-deoxyglucose quantitative autoradiography to explore the representation of direction in macaque FEF. They observed a mediolateral organization of saccade direction opposite to what is observed here in the marmoset, with the upper visual field represented ventrolaterally. Further investigation is required to see whether there exists a true species difference between marmosets and macaques in representation of saccade direction. Some variations in saccade direction and amplitude may exist across cortical layers. We were unable to address this here due to the fixed length of the electrodes in the arrays we used. 
At more posterior-medial sites where larger saccades are represented (dFEF), we observed skeletomotor responses resembling an orienting response while we only observed oculomotor responses at the more anterior-lateral sites. This is consistent with what Knight and Fuchs (2007) found in awake, headunrestrained macaques. Indeed, Foerster (1926) already reported two saccade-related fields in humans: (1) FEF where epileptic seizures evoked contralateral saccades and (2) a more posterior field that he termed frontal adversive field (frontales Adversivsfeld), where seizures were associated with contralateral saccades and head movements.

At posterior medial sites, at the border of area $6 \mathrm{D}$ and $6 \mathrm{M}$, we observed goal-directed saccades characteristic of SEF (Schlag and Schlag-Rey, 1987). Contrary to observations at more anterior lateral sites, convergence of saccades could not be explained by physical limitation of the orbit. We observed saccades converging at locations well within the animal's oculomotor range and, albeit infrequently, saccades directed to the hemifield ipsilateral to the stimulated hemisphere. These findings are similar to observations in the macaque by Schlag and Schlag-Rey (1987). However, we observed that saccade latencies were much longer at these sites (70-110 ms) than those observed by Schlag and Schlag-Rey (1987) (40-60 ms). Further, they observed low current thresholds, at many sites $<20 \mu \mathrm{A}$, whereas we observed few saccades at currents as high as $200 \mu \mathrm{A}$. Together, these findings suggest the observed responses may be evoked due to current spread to dorsomedial regions not covered by our arrays. We propose that area $6 \mathrm{M}$ may contain the putative marmoset SEF. Further investigation using ICMS and single unit electrophysiology in marmoset dorsomedial frontal cortex is required to fully investigate this putative homology.

We were also able to elicit saccades at rostral sites in area 46 and in anterior area $8 \mathrm{aD}$. At these sites, saccades were evoked at high currents and long latencies, and did not exhibit any clear organization of direction or amplitude. As with our observations in other areas of marmoset frontal cortex, this finding is consonant with previous work in macaque (Robinson and Fuchs, 1969). Further investigation in the frontal pole of the marmoset brain is required to characterize this region.

In a previous resting-state fMRI study, the FEF of marmoset was localized provisionally at the border of areas $8 \mathrm{aD}$ and $6 \mathrm{DR}$ based on the strength of functional connectivity between this region and the SC (Ghahremani et al., 2017). This finding appears at odds with previous task-based fMRI studies which observed robust visual and saccadic responses in areas $8 \mathrm{aV}$ (Hung et al., 2015; Schaeffer et al., 2019). More recent resting-state fMRI in awake marmosets may serve to clarify this discrepancy, with SC showing peaks of connectivity in both lateral $8 \mathrm{aV}$ and $8 \mathrm{aD} / 6 \mathrm{DR}$ (Schaeffer et al., 2019; Fig. 7). Indeed, isoflurane anesthesia has been shown to obfuscate the full extent of resting-state connectivity profiles (Hutchison et al., 2014). Here, we found that the characteristics of ICMS-evoked saccades in area $8 \mathrm{aV}$ were consistent with the classically defined FEF in macaque, whereas those in area $8 \mathrm{aD}$ were not. A potential explanation for this discrepancy may lie in the differences in the density and termination patterns of corticotectal projections between the FEF and more medial frontal areas. In macaques, it has been shown that the projections from area 6DR terminate in a more widespread area within the SC than those from FEF (Shook et al., 1990). If such a difference also exists in marmosets, it may account for the greater SC functional connectivity observed at the $6 \mathrm{DR} / 8 \mathrm{aD}$ border. From this account, both sets of findings can be reconciled, because the resting-state data would be consistent with the strength of ana- tomical connections, whereas evidence from functional studies using fMRI and ICMS point to a common locus of FEF. A definitive appraisal of such an explanation awaits more detailed anatomical investigations of the connectivity of the physiologically defined FEF and medial frontal cortex in the marmoset.

In a recently published study, we performed an electrophysiological investigation of preparatory activity for pro-saccades and anti-saccades, in which we recorded in area $8 \mathrm{aD}$ (Johnston et al., 2019), which we referred to as the putative FEF of the marmoset based on the strength of resting-state functional connectivity as discussed above (Ghahremani et al., 2017). In that work, we performed recordings with laminar electrodes that were inserted into the cortex daily at sites corresponding to approximately the border of areas $8 \mathrm{aD}$ and 6DR. As noted above, a more recent resting-state fMRI study has shown a peak of SC connectivity in this region, in addition to the more lateral area $8 \mathrm{aV}$. We found here that microstimulation evoked larger amplitude saccades from the region corresponding to area $8 \mathrm{aD} / 6 \mathrm{DR}$. Given the broad tuning of FEF neurons (Bruce et al., 1985), and the positioning of our stimuli on the horizontal meridian at an eccentricity of $\sim 7^{\circ}$, we believe that, although not optimized, our stimuli were within the response fields of saccade-related neurons in this area. We thus do not believe that our current results affect the interpretation of our findings there. Future work characterizing the tuning characteristics of these areas for visual stimuli and saccades should prove illuminating.

Altogether, our data demonstrate a similar functional organization of the FEF in marmosets and macaques and provide a combined physiological characterization and anatomical localization that opens avenues for future exploration of FEF microcircuitry in marmosets. Electrophysiological studies in marmosets have the potential to complement ongoing work in the macaque model and human participants by advancing our understanding of laminar processes and their contributions to the oculomotor and cognitive functions of this area.

\section{References}

Avants BB, Tustison NJ, Song G, Cook PA, Klein A, Gee JC (2011) A reproducible evaluation of ANTs similarity metric performance in brain image registration. Neuroimage 54:2033-2044.

Awh E, Armstrong KM, Moore T (2006) Visual and oculomotor selection: links, causes and implications for spatial attention. Trends Cogn Sci 10: $124-130$.

Blum B, Kulikowski JJ, Carden D, Harwood D (1982) Eye movements induced by electrical stimulation of the frontal eye fields of marmosets and squirrel monkeys. Brain Behav Evol 21:34-41.

Bruce CJ, Goldberg ME, Bushnell MC, Stanton GB (1985) Primate frontal eye fields. II. Physiological and anatomical correlates of electrically evoked eye movements. J Neurophysiol 54:714-734.

Burish MJ, Stepniewska I, Kaas JH (2008) Microstimulation and architectonics of frontoparietal cortex in common marmosets (Callithrix jacchus). J Comp Neurol 507:1151-1168.

Collins CE, Lyon DC, Kaas JH (2005) Distribution across cortical areas of neurons projecting to the superior colliculus in new world monkeys. Anat Rec 285A:619-627.

Corneil BD, Elsley JK, Nagy B, Cushing SL (2010) Motor output evoked by subsaccadic stimulation of primate frontal eye fields. Proc Natl Acad Sci U S A 107:6070-6075.

Elsley JK, Nagy B, Cushing SL, Corneil BD (2007) Widespread presaccadic recruitment of neck muscles by stimulation of the primate frontal eye fields. J Neurophysiol 98:1333-1354.

Ferrier D (1875) The Croonian lecture: experiments on the brain of monkeys (second series). Philos Trans R Soc Lond 165:433-488.

Foerster O (1926) Zur operativen behandlung der epilepsie. Dtsch Z Fur Nervenheilkd 89:137-147.

Ghahremani M, Hutchison RM, Menon RS, Everling S (2017) Frontopari- 
etal functional connectivity in the common marmoset. Cereb Cortex 27:3890-3905.

Gilbert KM, Schaeffer DJ, Gati JS, Klassen LM, Everling S, Menon RS (2019) Open-source hardware designs for MRI of mice, rats, and marmosets: integrated animal holders and radiofrequency coils. J Neurosci Methods 312:65-72.

Gottlieb JP, Bruce CJ, MacAvoy MG (1993) Smooth eye movements elicited by microstimulation in the primate frontal eye field. J Neurophysiol 69:786-799.

Heiney SA, Blazquez PM (2011) Behavioral responses of trained squirrel and rhesus monkeys during oculomotor tasks. Exp Brain Res 212: 409-416.

Hung CC, Yen CC, Ciuchta JL, Papoti D, Bock NA, Leopold DA, Silva AC (2015) Functional MRI of visual responses in the awake, behaving marmoset. Neuroimage 120:1-11.

Hutchison RM, Culham JC, Everling S, Flanagan JR, Gallivan JP (2014) Distinct and distributed functional connectivity patterns across cortex reflect the domain-specific constraints of object, face, scene, body, and tool category-selective modules in the ventral visual pathway. Neuroimage 96:216-236.

Johnston KD, Barker K, Schaeffer L, Schaeffer D, Everling S (2018) Methods for chair restraint and training of the common marmoset on oculomotor tasks. J Neurophysiol 119:1636-1646.

Johnston K, Ma L, Schaeffer L, Everling S (2019) Alpha oscillations modulate preparatory activity in marmoset area 8Ad. J Neurosci 39:1855-1866.

Knight TA, Fuchs AF (2007) Contribution of the frontal eye field to gaze shifts in the head-unrestrained monkey: effects of microstimulation. J Neurophysiol 97:618-634.

Li X, Morgan PS, Ashburner J, Smith J, Rorden C (2016) The first step for neuroimaging data analysis: DICOM to NIfTI conversion. J Neurosci Methods 264:47-56.

Liu C, Ye FQ, Yen CC, Newman JD, Glen D, Leopold DA, Silva AC (2018) A digital 3D atlas of the marmoset brain based on multi-modal MRI. Neuroimage 169:106-116.

Mitchell JF, Reynolds JH, Miller CT (2014) Active vision in marmosets: a model system for visual neuroscience. J Neurosci 34:1183-1194.

Mitchell JF, Leopold DA (2015) The marmoset monkey as a model for visual neuroscience. Neuroscience Research 93:20-46.

Mott FW, Schuster E, Halliburton WD (1910) Cortical lamination and localisation in the brain of the marmoset. Proceedings of the Royal Society of London. Series B, Containing Papers of a Biological Character (19051934) $82: 124-134$.
Peterson J, Chaddock R, Dalrymple B, Van Sas F, Gilbert KM, Klassen LM, Gati JS, Handler WB, Chronik BA (2018) Development of a gradient and shim insert system for marmoset imaging at 9.4 T. Paper presented at 26th Annual Meeting of International Society for Magnetic Resonance in Medicine, Paris, June.

Reser DH, Burman KJ, Yu H-H, Chaplin TA, Richardson KE, Worthy KH, Rosa MGP (2013) Contrasting Patterns of Cortical Input to Architectural Subdivisions of the Area 8 Complex: A Retrograde Tracing Study in Marmoset Monkeys. Cereb Cortex 23:1901-1922.

Robinson DA, Fuchs AF (1969) Eye movements evoked by stimulation of frontal eye fields. J Neurophysiol 32:637-648.

Russo GS, Bruce CJ (1993) Effect of eye position within the orbit on electrically elicited saccadic eye movements: a comparison of the macaque monkey's frontal and supplementary eye fields. J Neurophysiol 69:800-818.

Savaki HE, Gregoriou GG, Bakola S, Moschovakis AK (2015) Topography of visuomotor parameters in the frontal and premotor eye fields. Cereb Cortex 25:3095-3106.

Schaeffer DJ, Gilbert KM, Hori Y, Hayrynen LK, Johnston KD, Gati JS, Menon RS, Everling S (2019) Task-based fMRI of a free-viewing visuosaccadic network in the marmoset monkey. Neuroimage 202:116147.

Schall JD (1997) Visuomotor areas of the frontal lobe. In: Extrastriate cortex in primates (Rockland KS, Kaas JH, Peters A eds), pp 527-638. Boston: Springer.

Schlag J, Schlag-Rey M (1987) Evidence for a supplementary eye field. J Neurophysiol 57:179-200.

Shook BL, Schlag-Rey M, Schlag J (1990) Primate supplementary eye field: I. comparative aspects of mesencephalic and pontine connections. J Comp Neurol 301:618-642.

Smith SM, Jenkinson M, Woolrich MW, Beckmann CF, Behrens TE, Johansen-Berg H, Bannister PR, De Luca M, Drobnjak I, Flitney DE, Niazy RK, Saunders J, Vickers J, Zhang Y, De Stefano N, Brady JM, Matthews PM (2004) Advances in functional and structural MR image analysis and implementation as FSL. Neuroimage 23:S208-S219.

Stanton GB, Deng SY, Goldberg ME, McMullen NT (1989) Cytoarchitectural characteristic of the frontal eye fields in macaque monkeys. J Comp Neurol 282:415-427.

Tomlinson RD, Bahra PS (1986) Combined eye-head gaze shifts in the primate. I. metrics. J Neurophysiol 56:1542-1557.

Wakabayashi M, Koketsu D, Kondo H, Sato S, Ohara K, Polyakova Z, Chiken S, Hatanaka N, Nambu A (2018) Development of stereotaxic recording system for awake marmosets (Callithrix jacchus). Neurosci Res 135: 37-45. 